\title{
SPECTRUM AND FUNCTIONS OF OPERATORS ON DIRECT FAMILIES OF BANACH SPACES*
}

\author{
M. I. GIL ${ }^{\dagger}$
}

Abstract. We investigate the spectrum, resolvent and analytic functions of bounded linear operators on so called direct families of Banach spaces. The direct families of Banach spaces can be considered on the one hand as a particular generalization of the notion of the direct integral of Hilbert spaces introduced by von Neumann, and on the other hand as a generalization of Banach spaces with mixed norms. As it is shown in the paper, the direct families of Banach spaces is a natural tool for the investigation of partial integral operators of the type

$$
u \rightarrow w(x, y) u(x, y)+\int_{0}^{\phi(x)} K(x, y, s) u(x, s) d s(a \leq x \leq b ; 0 \leq y \leq \phi(x))
$$

where $w(.,$.$) and K(., .,$.$) and \phi$ are given functions. Partial integral operators play an essential role in numerous applications, in particular, in physics, mechanics, kinetic and transport theories, etc. We also discuss applications of our results to the Barbashin type integro-differential equations.

Key words. Banach space, linear operator, resolvent, spectrum, perturbation, direct families, mixed norms, partial integral operator, Barbashin type equation.

AMS subject classifications. 47A10, 47A56, 47G10, 47B10, 45P05

1. Definitions and preliminaries. Let $Z=Z(\omega)$ be a Banach space of real scalar valued functions defined on a locally compact set $\omega$ with a norm $|\cdot|_{Z}$. For any $x \in \omega$, let $H(x)$ be a Banach space with a norm $|\cdot|_{H(x)}$. Introduce the Banach space $X$ of mappings $f: x \in \omega \rightarrow H(x)$, such that $|f(x)|_{H(x)} \in Z(\omega)$, and $X$ is equipped with the norm

$$
|f|_{X}=\left.|| f(x)\right|_{H(x)} \mid Z
$$

Then we will call $X$ a direct family of spaces $H(x)$ with the basic space $Z(\omega)$ and write $X=X(Z(\omega), H()$.$) , and f=(f(x))_{x \in \omega}$.

For example, let $Z(\omega)=L^{p}(\omega) \quad(1 \leq p<\infty)$ be the space of functions defined on a set $\omega$ with the finite norm

$$
|v|_{Z}=|v|_{L^{p}}=\left[\int_{\omega}|v(x)|^{p} d x\right]^{1 / p} .
$$

Then $X=X\left(L^{p}(\omega), H().\right)$ and

$$
|f|_{X}=\left[\int_{\omega}|f(x)|_{H(x)}^{p} d x\right]^{1 / p}\left(f=(f(x))_{x \in \omega} ; f(x) \in H(x), x \in \omega\right) .
$$

Below we consider more concrete examples of space $X$.

In the present paper we investigate the spectrum, resolvent and analytic functions of bounded linear operators on the direct families of Banach spaces. The direct families of Banach spaces can be considered on the one hand as a partial generalization of the notion of the direct integral of Hilbert spaces introduced by von Neumann,

\footnotetext{
${ }^{*}$ Received July 3, 2009; accepted for publication March 3, 2010. This research was supported by the Kamea Fund of the Israel.

${ }^{\dagger}$ Department of Mathematics, Ben Gurion University of the Negev, P.0. Box 653, Beer-Sheva 84105, Israel (gilmi@cs.bgu.ac.il).
} 
cf. [19], and on the other hand as a generalization of Banach spaces with mixed norms, e.g. $[1,5]$. The direct families of Banach spaces is a natural tool for the investigation of partial integral operators discussed in Section 6 below. Partial integral operators play an essential role in numerous applications, in particular, in kinetic theory [16], in physics and mechanics [3]. About other applications see [4, 14, 18]. In contrast to the usual (ordinary) integral operators, partial integral operators have rather unpleasant properties: they are noncompact even if the kernels are continuous. This is probably the reason why the partial integral operators have not been studied yet as systematically as the ordinary integral operators. In the sequel $I$ is the unit operator in the corresponding space. For a linear operator $A, \sigma(A)$ is the spectrum; $\lambda_{k}(A), k=1,2, \ldots$ are the eigenvalues with their multiplicities and $R_{z}(A)=(A-z I)^{-1}$ $(z \notin \sigma(A))$ is the resolvent. By $M(Y)$ the set of bounded linear operators acting in a Banach space $Y$ is denoted.

For any $x \in \omega$, let $A(x)$ be a bounded linear operator in $H(x)$, such that the function $x \rightarrow A(x) f(x)$ is in $X$ for any $f \in X$. Introduce in $X$ the operator $\tilde{A}$ by

$$
(\tilde{A} f)(x)=A(x) f(x) \quad\left(f=(f(x))_{x \in \omega}\right) .
$$

Then we will call $\tilde{A}$ a direct family of operators $A(x)$ and write $\tilde{A}=(A(x))_{x \in \omega}$.

We need the following simple lemma.

Lemma 1.1. Let $A(x) \in M(H(x)), x \in \omega$, and

$$
\sup _{x \in \omega}|A(x)|_{H(x)}<\infty .
$$

Then $\tilde{A}=(A(x))_{x \in \omega}$ is a bounded operator in $X$, and

$$
|\tilde{A}|_{X} \leq \sup _{x \in \omega}|A(x)|_{H(x)} .
$$

Proof. Denote $w_{A}=\sup _{x \in \omega}|A(x)|_{H(x)}$. Then for a $f(.) \in X$ we have

$$
|\tilde{A} f(.)|_{X}=\left.\left.|| A(x) f(x)\right|_{H(x)}\left|Z \leq w_{A}\right||f(x)|_{H(x)}\left|Z=w_{A}\right| f(.)\right|_{X} .
$$

As claimed.

The previous lemma implies the following result.

Corollary 1.2. Let $A(x) \in M(H(x)), x \in \omega$, and for a $\lambda \in \mathbb{C}$, let the inequality

$$
\sup _{x \in \omega}\left|R_{\lambda}(A(x))\right|_{H(x)}<\infty
$$

hold. Then $\lambda$ is a regular point of $\tilde{A}$ and

$$
\left|R_{\lambda}(\tilde{A})\right|_{X} \leq \sup _{x \in \omega}\left|R_{\lambda}(A(x))\right|_{H(x)} .
$$


2. Spectral variations and operator functions. Let $A$ and $T$ be linear operators in an arbitrary Banach space $Y$. Then the quantity

$$
s v_{A}(T):=\sup _{\mu \in \sigma(T)} \inf _{\lambda \in \sigma(A)}|\mu-\lambda|
$$

is called the spectral variation of $T$ with respect to $A$.

Lemma 2.1. Let $\tilde{A}$ be defined by (1.2) and $T$ be a bounded linear operator in $X$. In addition, with the notation $\nu=|\tilde{A}-T|_{X}$, let the condition

$$
\nu \sup _{x \in \omega}\left|R_{z}(A(x))\right|_{H(x)}<1
$$

hold. Then $\lambda$ is a regular point of $T$. Therefore, for each $\mu \in \sigma(T)$ there is at least one $x \in \omega$, such that either $\mu \in \sigma(A(x))$, or

$$
\nu\left|R_{\mu}(A(x))\right|_{H(x)} \geq 1 .
$$

Proof. The required result is due to (1.4).

Let $\rho(A, \lambda)$ denote the distance between a point $\lambda \in \mathbb{C}$ and $\sigma(A)$ :

$$
\rho(A, \lambda):=\inf _{s \in \sigma(A)}|s-\lambda|
$$

Lemma 2.2. Let $\tilde{A}$ be defined by (1.2) and $T$ be a bounded linear operator in $X=X(Z(\omega), H()$.$) . In addition, let$

$$
\left|R_{\lambda}(A(x))\right|_{H(x)} \leq \Phi(x, 1 / \rho(A(x), \lambda)) \quad(\lambda \notin \sigma(A(x)) ; x \in \omega)
$$

where $\Phi(x, y)$ for each $x \in \omega$, is a continuous monotonically increasing non-negative function of a non-negative variable $y$, such that $\Phi(x, 0)=0$ and $\Phi(x, \infty)=\infty$. Then for any $\mu \in \sigma(T)$, there is at least one $x \in \omega$ and an $s \in \sigma(A(x))$, such that $|\mu-s| \leq z(x, \nu)$, where $z(x, \nu)$ is the unique positive root of the equation

$$
\nu \Phi(x, 1 / z)=1 .
$$

Proof. By (2.1)

$$
\nu \Phi\left(x, \rho^{-1}(A(x), \mu)\right) \geq 1 .
$$

Since $\Phi$ monotonically increases in $y, \rho(A(x), \mu) \leq z(x, \nu)$. This proves the lemma.

Let $X=X(Z(\omega), H()$.$) and \tilde{A}=(A(x))$ be defined by $(1.2)$, where $A(x) \quad(x \in \omega)$ is a bounded operator in $H(x)$. Let $F$ be a scalar-valued function, which is analytic on a neighborhood of $\sigma(A(x))$. Let a contour $C_{x}$ consist of a finite number of rectifiable Jordan curves, oriented in the positive sense customary in the theory of complex variables. Suppose that $C_{x}$ is contained in the domain of analyticity of $F$ and surrounds $\sigma(A(x))$ for all $x \in \omega$. Then we put

$$
F(A(x))=-\frac{1}{2 \pi i} \int_{C_{x}} F(\lambda) R_{\lambda}(A(x)) d \lambda
$$


and

$$
(F(\tilde{A}) f)(x)=F(A(x)) f(x), \quad\left(f=(f(x))_{x \in \omega}\right) .
$$

Suppose that

$$
\sup _{x \in \omega}|F(A(x))|_{H(x)}<\infty .
$$

Then by Lemma $1.1 F(\tilde{A})$ is a bounded operator in $X=X(Z(\omega), H()$.$) , and$

$$
|F(\tilde{A})|_{X} \leq \sup _{x \in \omega}|F(A(x))|_{H(x)} .
$$

3. Operators with nilpotent parts from ideals of Hilbert spaces. In the sequel, for each $x \in \omega, H(x)$ is a separable Hilbert space with a scalar product $(., .)_{H(x)}$ and the norm $|\cdot|_{H(x)}=\sqrt{(., .)_{H(x)}}$.

Furthermore, let $H$ be an arbitrary separable Hilbert space. A compact quasinilpotent operator will be called a Volterra operator, cf. [8]. Let $E(t)$ be a leftcontinuous orthogonal resolution of the identity in $H$, defined on a real segment $[a, b]$. $E$ is called a maximal resolution of the identity (m.r.i.), if its every gap $E\left(t_{0}+0\right)-E\left(t_{0}\right)$ (if it exists) is one-dimensional, cf. [8]. An m.r.i. $E(t)$ belongs to $A \in M(H)$ (or $A$ has an m.r.i. $E(t))$, if $E(t) A E(t)=A E(t)(t \in[a, b])$. We will say that $A \in M(H)$ is a E-triangular operator if it has an m.r.i. $E$ defined on $[a, b]$ and admits the representation

$$
A=D+V
$$

where $D$ is a normal operator and $V$ is a Volterra one, having the following properties:

$$
E(t) V E(t)=V E(t) \text { and } D E(t)=E(t) D(t \in[a, b]) .
$$

A $E$-triangular operator $A$ has the property

$$
\sigma(A)=\sigma(D),
$$

cf. [8, Lemma 7.5.1]. Each compact operator is E-triangular [12], each operator having the Schatten-von Neumann Hermitian component is E-triangular; for more details see [8, Chapter 7]. Note that the notion of the triangular representation of operators similar to the notion of E-triangular operator was introduced in [12].

Let $\tilde{A}$ be defined by (1.2). We will say $\tilde{A}$ is a locally triangular operator, if for all $x \in \omega, A(x)$ is a E-triangular operator with an m.r.i. $E_{x}(t)$ dependent on $x$, in general. Furthermore, let $W(x)=W(H(x))$ be a norm ideal of compact linear operators in $H(x)$. That is, $W(x)$ is algebraically a two-sided ideal, which is complete in an auxiliary norm $N_{W(x)}($.$) for which N_{W(x)}(C B)$ and $N(B C)_{W(x)}(B \in W(x), C \in$ $M(H(x)))$ are both dominated by $|C|_{H(x)} N_{W(x)}(B)$. In addition, suppose that there are positive numbers $\theta_{k}(k=1,2, \ldots)$, independent on $x$ with $\theta_{k}^{1 / k} \rightarrow 0$ as $k \rightarrow \infty$, such that for an arbitrary Volterra operator $V(x) \in W(x)$, the inequality

$$
\left|V^{k}(x)\right|_{H(x)} \leq \theta_{k} N_{W(x)}^{k}(V(x)) \quad(x \in \omega)
$$

holds. Below we show that the Schatten-von Neumann ideals are examples of ideals $W(x)$. 
THeOREM 3.1. Let $X$ be a direct family of separable Hilbert spaces $H(x), x \in \omega$, with an arbitrary basic space $Z$, and $\tilde{A}$ be defined by (1.2), where $A(x)$ are bounded E-triangular operators. Suppose that for each $x \in \omega$, the nilpotent part $V(x)$ of $A(x)$ belongs to a norm ideal $W(x)$ with the property (3.4). In addition, for a $\lambda \in \mathbb{C}$, let

$$
\psi(\lambda):=\sup _{x \in \omega} \sum_{k=0}^{\infty} \frac{\theta_{k} N_{W(x)}^{k}(V(x))}{\rho^{k+1}(A(x), \lambda)}<\infty .
$$

Then $\lambda$ is a regular point of $\tilde{A}$ and $\left|R_{\lambda}(\tilde{A})\right|_{X} \leq \psi(\lambda)$.

Proof. In this proof, for the brevity put $A(x)=A, D(x)=D, H=H(x)$, $W=W(x)$ and $V(x)=V$ with a fixed $x \in \omega$.

Let $\lambda$ be a regular point of the operator $D$. According to the triangular representation $A=D+V$, we obtain

$$
R_{\lambda}(A)=(D+V-\lambda I)^{-1}=R_{\lambda}(D)\left(I+V R_{\lambda}(D)\right)^{-1} .
$$

Operator $V R_{\lambda}(D)$ for a regular point $\lambda$ of the operator $D$ is a Volterra one due to Lemma 7.3.4 from [8]. Therefore,

$$
\left(I+V R_{\lambda}(D)\right)^{-1}=\sum_{k=0}^{\infty}\left(V R_{\lambda}(D)\right)^{k}(-1)^{k}
$$

and the series converges in the operator norm. Thus,

$$
R_{\lambda}(A)=R_{\lambda}(D) \sum_{k=0}^{\infty}\left(V R_{\lambda}(D)\right)^{k}(-1)^{k} .
$$

Since $V R_{\lambda}(D) \in Y$ is a Volterra operator, according to (3.4),

$$
\left|\left(V R_{\lambda}(D)\right)^{k}\right|_{H} \leq \theta_{k} N_{W}^{k}\left(V R_{\lambda}(D)\right) .
$$

But $N_{W}\left(V R_{\lambda}(D)\right) \leq N_{W}(V)\left|R_{\lambda}(D)\right|_{H}$, and thanks to (3.3), $\left|R_{\lambda}(D)\right|_{H}=\frac{1}{\rho(D, \lambda)}=$ $\frac{1}{\rho(A, \lambda)}$. So

$$
\left\|\left(V R_{\lambda}(D)\right)^{k}\right\| \leq \frac{\theta_{k}|V|_{Y}^{k}}{\rho^{k}(A, \lambda)}
$$

Relation (3.7) implies

$$
\left|R_{\lambda}(A)\right|_{H} \leq\left|R_{\lambda}(D)\right|_{H} \sum_{k=0}^{\infty}\left|\left(V R_{\lambda}(D)\right)^{k}\right|_{H} \leq \sum_{k=0}^{\infty} \frac{\theta_{k} N_{W}^{k}(V)}{\rho^{k+1}(A, \lambda)} .
$$

Now taking into account that $A=A(x)$, by Corollary 2.3 we get the required result.

4. Operators with variable Schatten-von Neumann nilpotent parts. Let $H$ be an arbitrary separable Hilbert space, again. For an integer $q=1,2, .$. , let $S_{2 q} \subset M(H)$ be the Schatten-von Neumann ideal with the finite ideal norm

$$
N_{2 q}(K):=\left[\operatorname{Trace}\left(K^{*} K\right)^{q}\right]^{1 / 2 q}\left(K \in S_{2 q}\right) .
$$


The asterisk means the adjoint. Let

$$
V \in S_{2 q}
$$

be a Volterra operator in $H$. Then due to Corollary 6.9.4 from [8],

$$
\left|V^{j}\right|_{H} \leq \theta_{j}^{(q)} N_{2 q}^{j}(V) \quad(j=1,2, \ldots)
$$

where

$$
\theta_{j}^{(q)}=\frac{1}{\sqrt{[j / q] !}}
$$

and $[x]$ means the integer part of a positive number $x$. Inequality (4.2) can be written as

$$
\left|V^{k q+m}\right|_{H} \leq \frac{N_{2 q}^{q k+m}(V)}{\sqrt{k !}}(k=0,1,2, \ldots ; m=0, \ldots, q-1) .
$$

In particular if $V \in S_{2}$, i.e. is a Hilbert-Schmidt operator, then

$$
\left|V^{k}\right|_{H} \leq \frac{N_{2}^{k}(V)}{\sqrt{k !}}(k=0,1,2, \ldots)
$$

Theorem 3.1 and (4.2) imply

Corollary 4.1. Let $\tilde{A}$ be defined by (1.2) where $A(x)$ are E-triangular operators. Let the nilpotent part $V(x)$ of $A(x), x \in \omega$, belong to $S_{2 q}(q=1,2, \ldots)$ and for $a \lambda \in \mathbb{C}$, let

$$
\hat{\rho}(\tilde{A}, \lambda):=\inf _{x \in \omega} \rho(A(x), \lambda)>0
$$

and

$$
v_{2 q}:=\sup _{x \in \omega} N_{2 q}(V(x))<\infty .
$$

Then $\lambda$ is a regular point of $\tilde{A}$, and

$$
\left|R_{\lambda}(\tilde{A})\right|_{X} \leq \sum_{k=0}^{\infty} \frac{\theta_{k}^{(q)} v_{2 q}^{k}}{\hat{\rho}^{k+1}(\tilde{A}, \lambda)} .
$$

If $V(x)$ is a Hilbert-Schmidt operator and (4.4), and (4.5) hold with $q=1$, then due to the previous corollary

$$
\left|R_{\lambda}(\tilde{A})\right|_{X} \leq \sum_{k=0}^{\infty} \frac{v_{2}^{k}}{\hat{\rho}^{k+1}(\tilde{A}, \lambda) \sqrt{k !}} \quad(\lambda \notin \sigma(\tilde{A})) .
$$

Note that inequality (4.6) is equivalent to the following one

$$
\left|R_{\lambda}(\tilde{A})\right|_{X} \leq \sum_{j=0}^{q-1} \sum_{k=0}^{\infty} \frac{v_{q}^{q k+j}}{\hat{\rho}^{q k+j+1}(\tilde{A}, \lambda) \sqrt{k !}} .
$$


Hence by the Schwarz inequality, we easily get

$$
\left|R_{\lambda}(\tilde{A})\right|_{X} \leq \sqrt{2} \sum_{j=0}^{q-1} \frac{v_{q}^{j}}{\hat{\rho}^{j+1}(\tilde{A}, \lambda)} \exp \left[\frac{v_{q}^{2 q}}{\hat{\rho}^{2 q}(\tilde{A}, \lambda)}\right] \quad(\lambda \notin \sigma(\tilde{A})) .
$$

Furthermore, let $A$ be a bounded E-triangular operator in an arbitrary separable Hilbert space $H$, again. Let its nilpotent part $V \in S_{2}$ and $F$ be a holomorphic function on a neighborhood of the closed convex hull co $(A)$ of the spectrum of $A$. Then by Theorem 6.9.1 [8],

$$
|F(A)|_{H} \leq \sum_{k=0}^{\infty} \sup _{\lambda \in \operatorname{co}(A)}\left|F^{(k)}(\lambda)\right| \frac{N_{2}^{k}(V)}{(k !)^{3 / 2}} .
$$

By this inequality and (2.5) we can assert the following result.

TheOREM 4.2. Let $\tilde{A}$ be a linear operator defined in $X(Z(\omega), H()$.$) by (1.2),$ where $A(x) \quad(x \in \omega)$ is a bounded E-triangular operator whose nilpotent part $V(x)$ is a Hilbert-Schmidt operator. In addition, for all $x \in \omega$, let function $F$ be holomorphic on a neighborhood of the closed convex hull co $(A(x))$ of the spectrum of $A(x)$, and

$$
\eta(F, \tilde{A}):=\sup _{x \in \omega} \sum_{k=0}^{\infty} \sup _{\lambda \in \operatorname{co}(A(x))}\left|F^{(k)}(\lambda)\right| \frac{N_{2}^{k}(V(x))}{(k !)^{3 / 2}}<\infty .
$$

Then $|F(\tilde{A})|_{X} \leq \eta(F, \tilde{A})$.

5. Variable compact and quasi-Hermitian operators. Let $A(x) \in S_{2} \quad(x \in$ $\omega)$. Then due to $[8$, Lemma 6.5 .2$]$

$$
N_{2}(V(x))=g(A(x))
$$

where

$$
g(A(x)):=\left(N_{2}^{2}(A(x))-\sum_{k=1}^{\infty}\left|\lambda_{k}(A(x))\right|^{2}\right)^{1 / 2} \leq N_{2}^{2}(A(x))-\mid \text { Trace } A^{2}(x) \mid .
$$

If $A(x)$ is a normal operator, then $g(A(x))=0$. If

$$
\sup _{x \in \omega} N_{2}(A(x))<\infty
$$

then

$$
\tilde{g}:=\sup _{x \in \omega} g(A(x))<\infty
$$

and under condition (4.4), inequality (4.7) implies

$$
\left|R_{\lambda}(\tilde{A})\right|_{X} \leq \frac{\sqrt{2}}{\hat{\rho}(\tilde{A}, \lambda)} \exp \left[\frac{\tilde{g}^{2}}{\hat{\rho}^{2}(\tilde{A}, \lambda)}\right] \quad(\lambda \notin \sigma(\tilde{A})) .
$$

Now let $A(x) \in S_{2 q}(x \in \omega ; q=2,3, \ldots)$. It is simple to check that $N_{2 q}(V(x)) \leq$ $2 N_{2 q}(A(x))$. Thus, if

$$
\hat{N}_{2 q}(\tilde{A}):=\sup _{x \in \omega} N_{2 q}(A(x))<\infty
$$


then (4.7) yields

$$
\left|R_{\lambda}(\tilde{A})\right|_{X} \leq \sqrt{2} \sum_{j=0}^{q-1} \frac{\left(2 \hat{N}_{2 q}(\tilde{A})\right)^{j}}{\hat{\rho}^{j+1}(\tilde{A}, \lambda)} \exp \left[\frac{\left(2 \hat{N}_{2 q}(\tilde{A})\right)^{2 q}}{\hat{\rho}^{2 q}(\tilde{A}, \lambda)}\right] \quad(\lambda \notin \sigma(\tilde{A})) .
$$

A linear operator in a Hilbert space is called a quasi-Hermitian operator if it is a sum of a selfadjoint operator and a compact one. Suppose that

$$
A_{I}(x):=\left(A(x)-A^{*}(x)\right) / 2 i \in S_{2} .
$$

Then due to [8, Lemma 7.7.2]

$$
N_{2}(V(x))=u(A(x))
$$

where

$$
u(A(x))=\sqrt{2}\left[N_{2}^{2}\left(A_{I}(x)\right)-\sum_{k=1}^{\infty}\left|\operatorname{Im} \lambda_{k}(A(x))\right|^{2}\right]^{1 / 2} \leq \sqrt{2} N_{2}\left(A_{I}(x)\right) .
$$

If $A(x)$ is a normal operator, then $u(A(x))=0$. Thus, if

$$
\sup _{x \in \omega} N_{2}\left(A_{I}(x)\right)<\infty
$$

then $\tilde{u}:=\sup _{x \in \omega} u(A(x))<\infty$, and under condition (4.4), according to (4.7), we get

$$
\left|R_{\lambda}(\tilde{A})\right|_{X} \leq \frac{\sqrt{2}}{\hat{\rho}(\tilde{A}, \lambda)} \exp \left[\frac{\tilde{u}^{2}}{\hat{\rho}^{2}(\tilde{A}, \lambda)}\right] \quad(\lambda \notin \sigma(\tilde{A})) .
$$

Similarly, by Lemmas 7.7.2 [8] and 7.15.2 [8] the cases $A^{*}(x)-A(x) \in S_{2 q}(q=2,3, \ldots)$ and

$$
A(x) A^{*}(x)-I \in S_{2 q}(q=1,2, \ldots)
$$

can be investigated.

Now let us turn to analytic functions. Theorem 4.2 and relation (5.1) imply

Corollary 5.1. Let $X=X(Z, H()$.$) and \tilde{A}$ be defined by (1.2), where $A(x) \quad(x \in \omega)$ is a Hilbert-Schmidt operator, satisfying condition (5.2). In addition, for all $x \in \omega$, let $F$ be holomorphic on a neighborhood of the closed convex hull $\operatorname{co}(A(x))$ of the spectrum of $A(x)$. If with the notation

$$
\psi_{k}:=\sup _{x \in \omega} \sup _{\lambda \in \operatorname{co}(A(x))} \frac{\left|F^{(k)}(\lambda)\right|}{(k !)^{3 / 2}},
$$

the condition

$$
\varlimsup_{k \rightarrow \infty} \sqrt[k]{\psi_{k}}<\frac{1}{\tilde{g}}
$$

holds, then

$$
|F(\tilde{A})|_{X} \leq \sum_{k=0}^{\infty} \psi_{k} \tilde{g}^{k}
$$


This result is sharp. Indeed, let $Z=L^{2}(\omega)$ and $A(x)$ be normal operators for all $x \in \omega$. Then $\tilde{A}$ is normal and $\tilde{g}=0$. Besides (5.7) becomes the equality

$$
|F(\tilde{A})|_{X}=\sup _{x \in \omega} \sup _{\lambda \in \sigma(A(x))}|F(\lambda)|,
$$

provided

$$
\sup _{\lambda \in \operatorname{co}(A(x))}|F(\lambda)|=\sup _{\lambda \in \sigma(A(x))}|F(\lambda)|
$$

for all $x \in \omega$. Similarly, Theorem 4.2 and relation (5.4) imply

Corollary 5.2. Let $X=X(Z, H()$.$) and \tilde{A}$ be defined by (1.2), where $A(x) \quad(x \in \omega)$ satisfies condition (5.5). In addition, for all $x \in \omega$, let $F$ be holomorphic on a neighborhood of co $(A(x))$ and the condition

$$
\varlimsup_{k \rightarrow \infty} \sqrt[k]{\psi_{k}}<\frac{1}{\tilde{u}}
$$

hold. Then

$$
|F(\tilde{A})|_{X} \leq \sum_{k=0}^{\infty} \psi_{k} \tilde{u}^{k}
$$

This result is also sharp. Namely, (5.10) becomes the equality (5.8), provided $A(x)$ is normal operators for all $x \in \omega$, and (5.9) holds.

EXAmple 5.3. Let condition (5.5) hold. Then

$$
\left|e^{\tilde{A} t}\right|_{X} \leq e^{\alpha(\tilde{A}) t} \sum_{k=0}^{\infty} \frac{t^{k} \tilde{u}^{k}}{(k !)^{3 / 2}} \quad(t \geq 0)
$$

where $\alpha(\tilde{A}):=\sup _{x} \operatorname{Re} \sigma(A(x))$.

6. Partial integral operators. For finite real numbers $a$ and $b>a$, let $\Lambda=$ $\{a \leq x \leq b, 0 \leq y \leq \phi(x)\}$, where $\phi(x)$ is a continuous nondecreasing positive function defined on $[a, b]$, and $X$ is the Banach space of complex functions $f(.,$.$) defined on \Lambda$ with the norm

$$
|f(., .)|_{X}=\left[\int_{a}^{b}\left(\int_{0}^{\phi(x)}|f(x, y)|^{2} d y\right)^{p / 2} d x\right]^{1 / p} \quad(1 \leq p<\infty) .
$$

Take $\omega=[a, b], H(x)=L^{2}(0, \phi(x))$ and $Z=L^{p}(a, b)$. So $X=\left(L^{p}(a, b), L^{2}(0, \phi()\right.$.$) .$ Besides,

$$
|v|_{H(x)}=\int_{0}^{\phi(x)}|v(y)|^{2} d y \quad\left(v \in L^{2}(0, \phi(x))\right) .
$$

Our main object in this section is the partial integral operator $\tilde{B}$ defined by

$$
(\tilde{B} f)(x, y)=w(x, y) f(x, y)+\int_{0}^{\phi(x)} K(x, y, s) f(x, s) d s
$$




$$
(a \leq x \leq b ; 0 \leq y \leq \phi(x) ; f \in X)
$$

where $w(x, y)$ and $K(x, y, s)$ are scalar functions satisfying the conditions pointed below. We can write

$$
(\tilde{B} f)(x, y)=B(x) f(x, y)
$$

where

$$
(B(x) v)(y)=w(x, y) v(y)+\int_{0}^{\phi(x)} K(x, y, s) v(s) d s \quad\left(v \in L^{2}(0, \phi(x)) .\right.
$$

So (1.2) holds. Operator $\tilde{B}$ is called a partial integral operator, inasmuch as the integration is carried out only with respect to some arguments, while the other arguments of the integrand are "frozen". The partial integral operators were investigated in the well-known papers [13] and [15]. In the paper [13] the author gives us sufficient conditions under which the spectral radius of a partial integral operator of Volterra type is zero, or a partial integral operator of Volterra-Fredholm type has a trivial essential spectrum. In the paper [15], interesting results on the existence, continuity and the regularity of solutions of the equations with partial integral operators are given, in particular applications to a linear integral equation, occurring in the mechanics of continuous media, are discussed. Note that in the pointed literature mainly the case $\phi(x) \equiv$ const is investigated. The notion of the direct family of Banach spaces enables us to apply many results on compact operators to partial integral operators. We consider the case of two variables only for simplicity. The suggested approach allows us to explore the operators acting in spaces of functions of several variables. Note that the special case when $Z$ is a Hilbert space was explored in [11].

Assume that $w(x, y)$ is measurable, real and bounded on $\Lambda$, and $K$ satisfies the condition

$$
\tilde{N}_{2}\left(K_{I}\right):=\frac{1}{2} \sup _{a \leq x \leq b}\left[\int_{a}^{b} \int_{0}^{\phi(x)}|K(x, y, s)-\bar{K}(x, s, y)|^{2} d s d y\right]^{1 / 2}<\infty .
$$

Since $w$ is real, the operator $B(x)$ satisfies the condition

$$
\sup _{a \leq x \leq b} N_{2}\left(B_{I}(x)\right)=\tilde{N}_{2}\left(K_{I}\right) \quad\left(B_{I}=\left(B-B^{*}\right) / 2 i\right) .
$$

Inequality (5.6) implies our next result.

THEOREM 6.1. Let $\tilde{B}$ be the partial integral operator defined by (6.2) and condition (6.3) hold. In addition, for $a \lambda \in \mathbb{C}$, let

$$
\hat{\rho}(\tilde{B}, \lambda):=\inf _{x \in[a, b]} \rho(B(x), \lambda)>0 .
$$

Then

$$
\left|R_{\lambda}(\tilde{B})\right|_{X} \leq \frac{\sqrt{2}}{\hat{\rho}(\tilde{B}, \lambda)} \exp \left[\frac{2 \tilde{N}_{2}^{2}\left(K_{I}\right)}{\hat{\rho}^{2}(\tilde{B}, \lambda)}\right] \quad(\lambda \notin \sigma(\tilde{B})) .
$$

Now we are going to establish bounds for spectra of the considered partial integral operator. Let

$$
v_{-}:=\left[\sup _{x \in[a, b]} \int_{0}^{\phi(x)} \int_{0}^{y}|K(x, y, s)|^{2} d s d y\right]^{1 / 2}<\infty
$$


and

$$
v_{+}:=\left[\sup _{x \in[a, b]} \int_{0}^{\phi(x)} \int_{y}^{\phi(x)}|K(x, y, s)|^{2} d s d y\right]^{1 / 2}<\infty
$$

Theorem 6.2. Let $\tilde{B}$ be the partial integral operator defined by (6.2). Then the spectrum of $\tilde{B}$ is included in the set

$$
\left\{z \in \mathbb{C}:|w(x, y)-z| \leq r(K) \leq \psi_{K}, \quad(x, y) \in \Lambda\right\},
$$

where $r(K)$ is the unique positive root of the equation

$$
1=v_{-} \frac{\sqrt{2}}{z} \exp \left[\frac{v_{+}^{2}}{z^{2}}\right]
$$

and

$$
\psi_{K}:=\left\{\begin{array}{ll}
e / a & \text { if } a \leq e \\
{[\ln (a)]^{-1 / 2}} & \text { if } a>e
\end{array} \text { with the notation } a=\frac{e^{1 / 2}}{\sqrt{2} v_{-}} .\right.
$$

Proof. Define the operators $D(x), V_{+}(x)$ and $V_{-}(x)$ by

$$
(D(x) v)(y)=w(x, y) v(y),\left(V_{+}(x) v\right)(y)=\int_{y}^{\phi(x)} K(x, y, s) v(s) d s,
$$

and

$$
\left(V_{-}(x) v\right)(y)=\int_{0}^{y} K(x, y, s) v(s) d s \quad\left(v \in L^{2}(0, \phi(x)) .\right.
$$

Put $B_{+}(x)=D(x)+V_{+}(x)$. Then the operator $\tilde{B}_{+}=\left(B_{+}(x)\right)_{x \in[a, b]}$ is locally Etriangular. Now apply Lemma 2.2 with $T=\tilde{B}$ and $\tilde{A}=\tilde{B}_{+}$. Then by Theorem 6.1 , for any $\mu \in \sigma(\tilde{B})$ we have

$$
|w(x, y)-\mu| \leq r(K) \quad(a \leq x \leq b ; 0 \leq y \leq \phi(x)) .
$$

But by Lemma 8.3.2 from [8], the inequality $r(K) \leq \psi_{K}$ holds. This proves the required result.

Certainly, in the previous theorem, one can exchange the places of $v_{-}$and $v_{+}$. By the previous theorem, the spectral radius of $\tilde{B}$ satisfies the inequality

$$
r_{s}(\tilde{B}) \leq \sup _{x, y \in \Lambda}|w(x, y)|+\psi_{K} .
$$

In particular, if $w(x, y) \equiv 0$, then $r_{s}(\tilde{B}) \leq \psi_{K}$. This inequality, in the case of the operators which are "close" to Volterra ones, is a refinement of the well-known estimate

$$
r_{s}(\tilde{B}) \leq \operatorname{vrai} \sup _{x, y} \int_{0}^{\phi(x)}|K(x, y, s)| d s,
$$

cf. [17, Section 16.6]. Note that the "inner" bounds for the spectrum of operator $\tilde{B}$ can be derived by making use the results of the papers [7,9]. Furthermore, we will 
say that a bounded linear operator $A$ is stable if $\operatorname{Re} \sigma(A)<0$. By Theorem 6.2 , we have

$$
\alpha(\tilde{B}):=\operatorname{Re} \sigma(\tilde{B}) \leq \sup _{x, y \in \Lambda} w(x, y)+\psi_{K} .
$$

So $\tilde{B}$ is stable, provided $\sup _{x, y \in \Lambda} w(x, y)<-\psi_{K}$.

Furthermore, Theorem 6.1 and Theorem 3.1 imply

Corollary 6.3. Let $\tilde{B}$ be defined by (6.2) and condition (6.3) hold. Let $T$ be a bounded linear operator acting in $X=X\left(L^{p}(a, b), L^{2}(0, \phi()).\right)(1 \leq p<\infty)$. Then for any $\mu \in \sigma(T)$, there are an $x \in[a, b]$ and an $s \in \sigma(B(x))$, such that $|\mu-s| \leq r(K, T)$, where $r(K, T)$ is the unique positive root of the equation

$$
1=|\tilde{B}-T|_{X} \frac{\sqrt{2}}{z} \exp \left[\frac{2 \tilde{N}_{2}^{2}\left(K_{I}\right)}{z^{2}}\right] .
$$

Note that $z(K, T)$ can be estimated by bounds for the zeros of entire functions suggested in [10].

7. The Barbashin type equations. Let $X, w(x, y)$ and $\phi(x)$ be the same as in the previous section. In this section we will consider the scalar Barbashin type equation

$$
\frac{\partial u(t, x, y)}{\partial t}=w(x, y) u(t, x, y)+\int_{0}^{\phi(x)} K(x, y, s) u(t, x, s) d s+\Psi(t, x, y)
$$

$$
(x, y \in \Lambda ; t \geq 0)
$$

where $\Psi(t, .,.) \in X, t \geq 0$.

The integro-differential equations of Barbashin type, naturally arise from systems of $n$ differential equations after passing to the limit $n \rightarrow \infty$, or after replacing discrete indices by continuous variables. Indeed, following [4, p. 431], consider, the system

$$
\frac{d q}{d t}=M q(t)+S u(t), z(t)=C q(t)+D u(t),
$$

where the values of $M, S, C$, and $D$ are $m \times m$-matrices, and the values of $q, z$, and $u$ are $m \times n$-matrices. That system represents the nonstationary balance equations for certain quantities (called substances) over given balance spaces, and the state vector $q$ represents a distribution density function for these substances. Physically, $n$ denotes the number of independent balance spaces, while $m$ is the number of independent substances. Here passing to the limit $n \rightarrow \infty$ has a natural physical meaning. In fact, $n \rightarrow \infty$ means that no more discrete balance spaces can be distinguished which occurs in systems with spatially distributed parameters. The mathematical model leads here to a system of $m$ partial differential equations. On the other hand, $m \rightarrow \infty$ means that one has to deal with an arbitrarily large number of substances. In this case we get the equation of the type

$$
\frac{\partial q(s, t)}{\partial t}=\gamma(s, t) q(s, t)+\int_{c}^{d} M_{0}(s, v) q(v, t) d v+\psi(s, t)
$$


where $\gamma(s, t)$ and $M_{0}(s, v)$ are matrix valued functions and $\psi(s, t)$ is a vector valued function. Equation (7.3) is an example of Barbashin type integro-differential equation with respect to $q(s, t)$. The variable $s \in[c, d]$ may have different meanings, according to the physical setting. More general example of Barbashin type integro-differential equation is given by the equation

$$
\frac{\partial q(s, x, t)}{\partial t}=\gamma(s, x, t) q(s, x, t)+\int_{c(x)}^{d(x)} M_{0}(s, x, v) q(v, x, t) d v+\psi(s, x, t)
$$

with a parameter $x$. For more details about the theory, history and applications of the Barbashin type equations see [4] and [18].

Furthermore, let us turn to equation (7.1). It is equivalent to the following one:

$$
u(t)=e^{\tilde{B} t} u(0)+\int_{0}^{t} e^{\tilde{B}(t-s)} \Psi(s) d s \quad(u(t)=u(t, x, y), \Psi(t)=\Psi(t, x, y))
$$

where the operator $\tilde{B}$ is defined as in the previous section. Take into account that (5.11) and (6.3) imply

$$
\left|e^{\tilde{B} t}\right|_{X} \leq \zeta(t) \quad(t \geq 0)
$$

where

$$
\zeta(t):=e^{\alpha(\tilde{B}) t} \sum_{k=0}^{\infty} \frac{\left(\sqrt{2} \tilde{N}_{2}\left(K_{I}\right) t\right)^{k}}{(k !)^{3 / 2}}
$$

Thus, for a solution $u(t)$ to equation (7.1), we get the following estimate:

$$
|u(t)|_{X} \leq \zeta(t)|u(0)|_{X}+\int_{0}^{t} \zeta(t-s)|\Psi(s)|_{X} d s .
$$

This inequality enables us to explore linear and nonlinear perturbations of the considered equations. To the best of our knowledge, such estimates are unknown in the available literature.

\section{REFERENCES}

[1] M. J. Ablowitz And H. Segur, Solitons and the Inverse Scattering Transform, SIAM Studies in Applied Mathematics, SIAM - Philadelphia, 1981.

[2] Y. A. Abramovich and C. D. Aliprantis, Problems in Operator Theory, Amer. Math. Soc., Providence, R.I., 2002.

[3] V. M. Aleksandrov and E. V. Kovalenko, Problems in Continuous Mechanics with Mixed Boundary Conditions, Nauka, Moscow 1986, in Russian.

[4] J. Appel, A. Kalitvin and P. Zabreiko, Partial Integral Operators and Integrodifferential Equations, Marcel Dekker, New York, 2000.

[5] A. Benedek And R. Pansone, The spaces with mixed norms, Duke Mat. J., 28 (1961), pp. 301324.

[6] M. C. Cercignani, Mathematical Methods in Kinetic Theory, Macmillian, New York, 1969.

[7] M. I. GIL', Invertibility conditions and bounds for spectra of matrix integral operators, Monatshefte für Mathematik, 129 (2000), pp. 15-24.

[8] M. I. GIL', Operator Functions and Localization of Spectra, Lecture Notes In Mathematics vol. 1830, Springer-Verlag, Berlin, 2003.

[9] M. I. GiL', Inner bounds for spectra of linear operators, Proc. Amer. Math. Soc., 131 (2003), pp. 3737-3746. 
[10] M. I. GIL', Bounds for zeros of entire functions, Acta Applicandae Mathematicae, 99:2 (2007), pp. 117-159.

[11] M. I. GIL', Spectrum and resolvent of a partial integral operator, Applicable Analysis, 87:5 (2008), pp. 555-566.

[12] I. C. Gohberg And M. G. Krein, Introduction to the Theory of Linear Nonselfadjoint Operators, Trans. Mathem. Monographs, v. 18, Amer. Math. Soc., R.I., 1969.

[13] A. S. Kalitvin, Spectral properties of partial integral operators of Volterra and VolterraFredholm type, Z. Anal. Anwend., 17:2 (1998), pp. 297-309.

[14] A. S. Kalitvin, On two problems for the Barbashin integro-differential equation, J. Math. Sci., New York, 126:6 (2005), pp. 1600-1606.

[15] A. S. Kalitvin, Zabrejko, P.P. On the theory of partial integral operators, J. Integral Equations Appl., 3:3 (1991), pp. 351-382.

[16] H. G. Kaper, C. G. LekKerkerker and J. Hejtmanek, Spectral Methods in Linear Transport Theory, Birkhauser, Basel 1982.

[17] M. A. Krasnosel'skit, J. Lifshits and A. Sobolev, Positive Linear Systems. The Method of Positive Operators, Heldermann Verlag, Berlin, 1989.

[18] A. D. Myshkis, Mixed functional differential equations, Contemporary Mathematics. Fundamental Directions, 4 (2003), pp. 5-120. In Russian.

[19] B. Scarpellini, Stability, Instability and Direct Integrals, Chapman and Hall, Boca Ration, 1999. 